\title{
Comparison of the effects of rolling resistance and angularity in sheared granular media
}

\author{
Nicolas Estrada*, Emilien Azéma ${ }^{\dagger}$, Farhang Radjai ${ }^{\dagger}$ and Alfredo Taboada** \\ *Departamento de Ingeniería Civil y Ambiental, Universidad de los Andes, Bogotá, Colombia \\ ${ }^{\dagger}$ LMGC,Université Montpellier 2 - CNRS, Montpellier, France \\ ** Laboratoire Géosciences Montpellier, Université Montpellier 2 - CNRS, Montpellier, France
}

\begin{abstract}
In this paper, we compare the effect of rolling resistance at the contacts in granular systems composed of disks with the effect of angularity in granular systems composed of regular polygonal particles. For this purpose, we use contact dynamics simulations. By means of a simple shear numerical device, we investigate the mechanical behavior of these materials in the steady state in terms of shear strength, solid fraction, force and fabric anisotropies, and probability distribution of contact forces. We find that, based on the energy dissipation associated with relative rotation between two particles in contact, the effect of rolling resistance can explicitly be identified with that of the number of sides in a regular polygonal particle. This finding supports the use of rolling resistance as a shape parameter accounting for particle angularity and shows unambiguously that one of the main influencing factors behind the mechanical behavior of granular systems composed of noncircular particles is the partial hindrance of rotations as a result of angular particle shape.
\end{abstract}

Keywords: Granular material, rolling resistance, angularity, shear strength, solid fraction, anisotropy, and force distribution. PACS: $45.70 . Đ n, 45.50 . Đ j, 83.10 . R s, 83.80 . \mathrm{Fg}$

\section{INTRODUCTION}

Real granular materials are rarely composed of spherical particles, and it has been shown that the nonspherical shape of the grains strongly influences the mechanical behavior of granular systems. The effect of grain shape is thus a crucial aspect to be taken into account for a realistic description of granular systems.

One of the numerical "tricks" that can be used to obtain realistic values of strength and solid fraction while using only circular particles in simulations is to partially restrict the relative rotations between grains [1]. For example, several studies have shown that rolling resistance leads to shear strengths and solid fractions that are comparable to those observed in granular soils and rocks, e.g., $[2,3,4,5]$. However, the extent to which rolling resistance can actually be compared to angular shape in more general terms, or whether rolling resistance and angular shape lead to similar structures at the mesoscopic scale, are interesting issues that remain poorly understood.

In this article, we compare, by means of discrete element simulations, the effects of rolling resistance and angularity. We construct two sets of polydisperse 2D packings. In the first set, the packings are composed of disks with an increasing magnitude of rolling resistance, whereas in the second set, the packings are composed of regular polygonal particles of increasing number of sides. By comparing various properties extracted from the two sets, we find a remarkable matching of the data from the disk packings with those of the polygon pack- ings for a rolling resistance expressed by a simple equation as a function of the number of sides. This one-to-one mapping between the two sets is based on energy dissipation considerations and might be generalized to other particle shapes. For more details about this work, see [6].

\section{MODEL DESCRIPTION}

All packings are made up of 7500 grains with diameters uniformly distributed by volume fractions between $0.6 d$ and $2.4 d$, where $d$ is the mean diameter. In all simulations, the coefficient of sliding friction $\mu_{s}$ between particles is 0.4 and collisions are perfectly inelastic. The particles are initially placed in a semiperiodic box $100 d$ wide using a geometrical procedure [7]. Next, the packing is sheared by imposing a constant shear velocity and a constant confining stress. To avoid strain localization at the boundaries, sliding and rolling are inhibited for the particles in contact with the walls. The samples are sheared up to a large cumulative shear strain $\gamma=\Delta x / h=5$, where $\Delta x$ is the horizontal displacement of the upper wall and $h$ is the thickness of the sample. All measures are averaged over the last $50 \%$ of cumulative shear strain in order to guarantee that they characterize the behavior of the system in the steady state, also known as the "critical state" in soil mechanics. In all tests, the gravity is set to zero.

The simulations were carried out by means of the contact dynamics method $[8,9,10,11]$, which assumes perfectly rigid particles interacting through mutual exclusion and Coulomb friction. For specific implementation 
of the contact dynamics method see [7, 11].

In the first set of samples, composed of disks, the rolling resistance is introduced through a rolling friction law [12], analogous to the sliding friction law. This law assumes that a contact can transmit a torque $M$ not exceeding a limit value $M_{\max }=\mu_{r} \ell f_{n}$, where $\mu_{r}$ is the coefficient of rolling friction, $\ell$ is the magnitude of the branch vector joining the centers of the contacting particles, and $f_{n}$ is the normal force. Relative rotation between two grains in contact is allowed only if $M=M_{\max }$.

In the second set of samples, composed of regular polygonal particles, two types of contact may occur: (1) between a corner and a side, and (2) between two sides. Side/side interactions represent two constraints and are treated by associating two contact points along the common side and applying the volume exclusion and the sliding friction law to each of them. Thus, in practice, two contact forces are calculated at each side/side contact. However, only their resultant and application point are physically relevant, and the result is independent of the choice of the two contact points [13]. Polygonal particles are simulated with the LMGC90 platform developed in Montpellier by F. Dubois and M. Jean.

\section{RESULTS}

The stress components can be calculated from the simulation data by the relation $\sigma_{i j}=n_{c}\left\langle f_{i}^{c} \ell_{j}^{c}\right\rangle$, where $n_{c}$ is the number of contacts per unit volume and the average runs over the contacts $c$ with contact force $f^{c}$ and branch vector $\ell^{c}$ [14]. The mean stress is $p=\left(\sigma_{1}+\sigma_{2}\right) / 2$, where $\sigma_{1}$ and $\sigma_{2}$ are the principal stress values, and the deviatoric stress is $q=\left(\sigma_{1}-\sigma_{2}\right) / 2$.

Figure 1 shows the shear strength $q / p$ and solid fraction $v=V_{p} / V$, where $V_{p}$ is the volume occupied by the particles and $V$ is the total volume, as functions of $\mu_{r}$ for the disks and of $1 / n_{s}$ for the polygons, where $n_{s}$ is the number of sides of the polygons. It can be seen that both $q / p$ and $v$ follow similar trends in the two sets as $\mu_{r}$ and $1 / n_{s}$ increase. However, a direct comparison of the data between the two sets is not possible in this representation due to the different physical meanings of $\mu_{r}$ and $1 / n_{s}$.

The respective effects of rolling friction and angular shape can be compared by their roles in the hindering of relative rotation. Let us consider a particle (a disk with rolling friction and a regular polygon) that rolls on a horizontal plane with a vertical force $N$ exerted at its center of mass and that is not allowed to slide. The work needed to displace the particle a distance equal to its perimeter is

$$
W_{d}=4 \pi \mu_{r} R_{d} N
$$

for the disk with rolling friction, where $R_{d}$ is the radius of the disk and the magnitude of the branch vector $\ell$

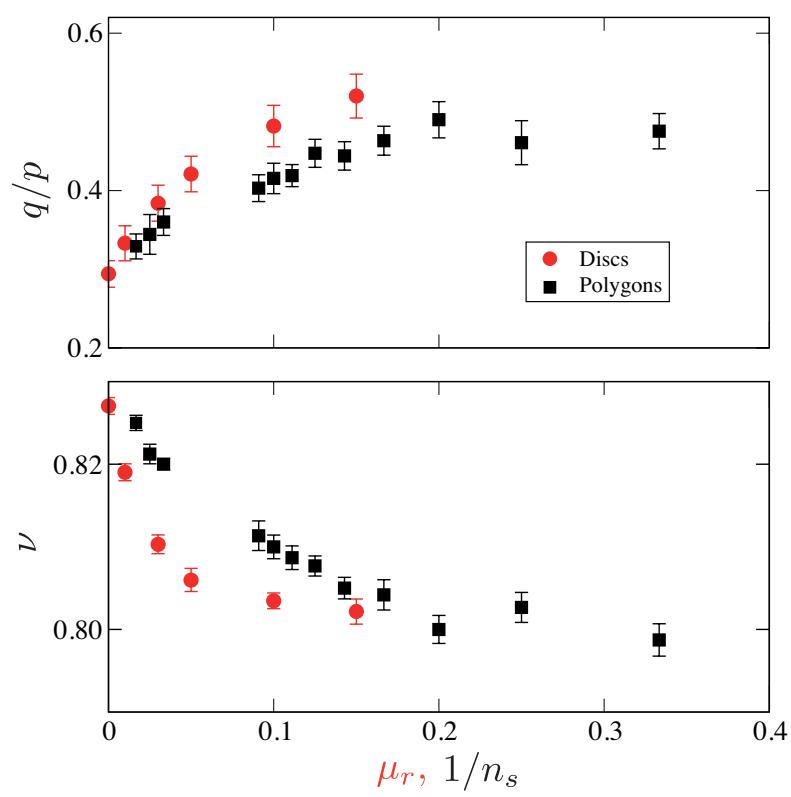

FIGURE 1. Shear strength $q / p$ (Up) and solid fraction $v$ (Down) as functions of $\mu_{r}$ for the disks and of $1 / n_{s}$ for the polygons. Error bars indicate the standard deviation.

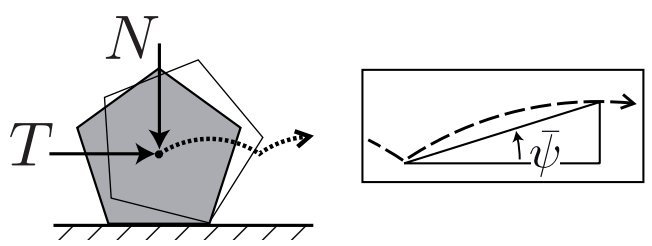

FIGURE 2. Trajectory of the center of mass of the polygon (dashed line) and definition of the mean dilatancy angle $\bar{\psi}$.

(necessary to calculate $M_{\max }$ ) has been replaced by the disk diameter, and

$$
W_{p}=n_{s}\left(1-\cos \left(\pi / n_{s}\right)\right) R_{p} N
$$

for the polygon, where $R_{p}$ is the radius of its circumcircle. Assuming equal work, i.e. $W_{d}=W_{p}$, we arrive at the following mapping between $\mu_{r}$ and $n_{s}$

$$
\mu_{r}=(1 / 4) \tan \bar{\psi}
$$

where it has been assumed that both particles have the same perimeter (i.e., $R_{p}=R_{d}\left(\pi / n_{s}\right) / \sin \left(\pi / n_{s}\right)$ ), and $\bar{\psi}=\pi /\left(2 n_{s}\right)$ is the mean dilatancy angle of the trajectory of the center of mass of the polygon (see Fig. 2). For a similar attempt to quantify the role of grain shapes in hindering relative rotation, see [15].

Figure 3 shows the shear strength $q / p$ and solid fraction $v$ as functions of $\mu_{r}$ for the disks and of $(1 / 4) \tan \bar{\psi}$ for the polygons. Remarkably, the shear strengths and solid fractions of the two sets of packings collapse, 

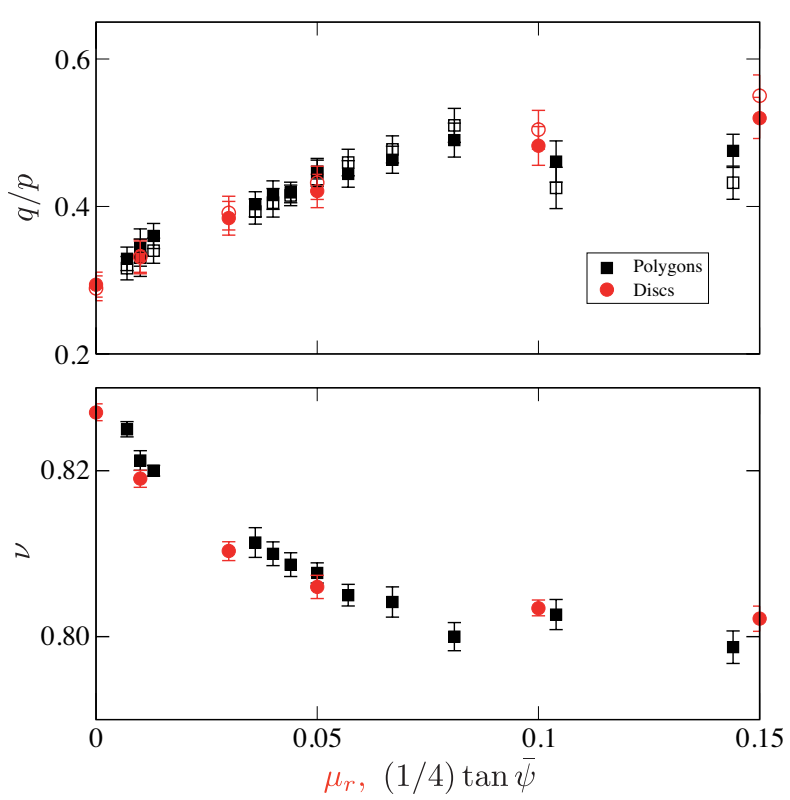

FIGURE 3. (Up) Shear strength $q / p$ as a function of $\mu_{r}$ for the disks and of $(1 / 4) \tan \bar{\psi}$ for the polygons, both from raw simulation data (full symbols) and as predicted by Eq. 4 (empty symbols). (Down) Solid fraction $v$ as a function of $\mu_{r}$ for the disks and of $(1 / 4) \tan \bar{\psi}$ for the polygons. Error bars indicate the standard deviation.

both increasing and decreasing, respectively, with $\mu_{r}$ and $(1 / 4) \tan \bar{\psi}$ and tending to a constant value at $\mu_{r}=$ $(1 / 4) \tan \bar{\psi} \simeq 0.1$. In other words, from a macro-scale viewpoint, a packing of regular polygons of $n_{s}$ sides is equivalent to a packing of disks with a coefficient of rolling friction $\mu_{r}$ given by Eq. 3. This result supports also the choice of the required energy for rolling as a relevant physical quantity for the rheology of granular materials.

The mapping evidenced in Fig. 3 hints at similar packing structures in the two sets. Figure 4 shows two snapshots: one representing a disk packing with $\mu_{r}=0.05$ and the other representing a polygon packing with $n_{s}=8$ (note that $0.05 \simeq(1 / 4) \tan (\pi /(2 * 8))$ according to Eq. $3)$. The contact forces are represented by segments joining the particle centers, with a thickness proportional to the force magnitude. We observe that the force-carrying backbone is astonishingly similar in the two systems.

From the expression of the stress tensor, it can be shown that the shear stress $q / p$ reflects the packing structure and force transmission via a simple relation [16]:

$$
q / p \simeq(1 / 2)\left(a_{c}+a_{n}+a_{t}\right),
$$

where $a_{c}, a_{n}$, and $a_{t}$, are the anisotropies of the angular distributions of contact orientations $P_{n}(\theta)$, normal forces $\left\langle f_{n}\right\rangle(\theta)$, and tangential forces $\left\langle f_{t}\right\rangle(\theta)$, respectively, as a function of contact orientation $\theta$, which are approxi-
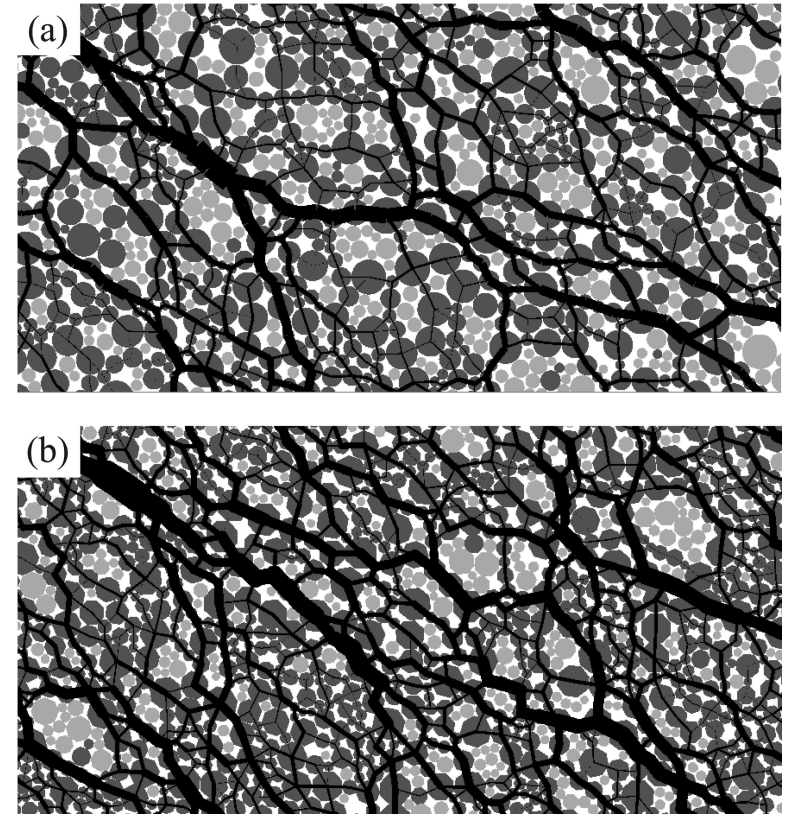

FIGURE 4. Snapshots of the force network in (a) a system composed of disks with rolling friction $\left(\mu_{r}=0.05\right)$ and (b) a system composed of octagonal particles. The line thickness is proportional to the normal force. The floating particles are represented in light grey.

mated by their lowest order Fourier expansions:

$$
\begin{aligned}
P_{n}(\theta) & \simeq 1 / \pi\left\{1+a_{c} \cos 2\left(\theta-\theta_{c}\right)\right\} \\
\left\langle f_{n}\right\rangle(\theta) & \simeq\left\langle f_{n}\right\rangle\left\{1+a_{n} \cos 2\left(\theta-\theta_{n}\right)\right\}, \\
\left\langle f_{t}\right\rangle(\theta) & \simeq-\left\langle f_{n}\right\rangle a_{t} \sin \left(\theta-\theta_{t}\right),
\end{aligned}
$$

where $\left\langle f_{n}\right\rangle$ is the mean normal fore, and $\theta_{c}=\theta_{n}=\theta_{t}$ are the corresponding privileged directions, which, in the steady state, coincide with the principal stress direction. Equation 4 reveals distinct origins of the shear strength in terms of force and texture anisotropy. The empty symbols in Fig. 3 show $q / p$ as predicted by Eq. 4 . We see that this equation approximates well the shear strength for all raw data.

The anisotropies $a_{c}, a_{n}$, and $a_{t}$ are shown in Fig. 5 as functions of $\mu_{r}$ for the disks and of $(1 / 4) \tan \bar{\psi}$ for the polygons. It is remarkable that all anisotropies are almost identical between the two sets. This correspondence is only broken for polygons with small numbers of sides, i.e., for $n_{s}=3$ and 4 . This happens because for these polygons the contact orientation is strongly influenced by the low rotational symmetry of the particles and the orientations of the sides rather than the relative positions of the particles.

As shown in [6], the mapping between rolling friction and angular shape of particles is also reflected by the probability density function (PDF) of normal forces. 

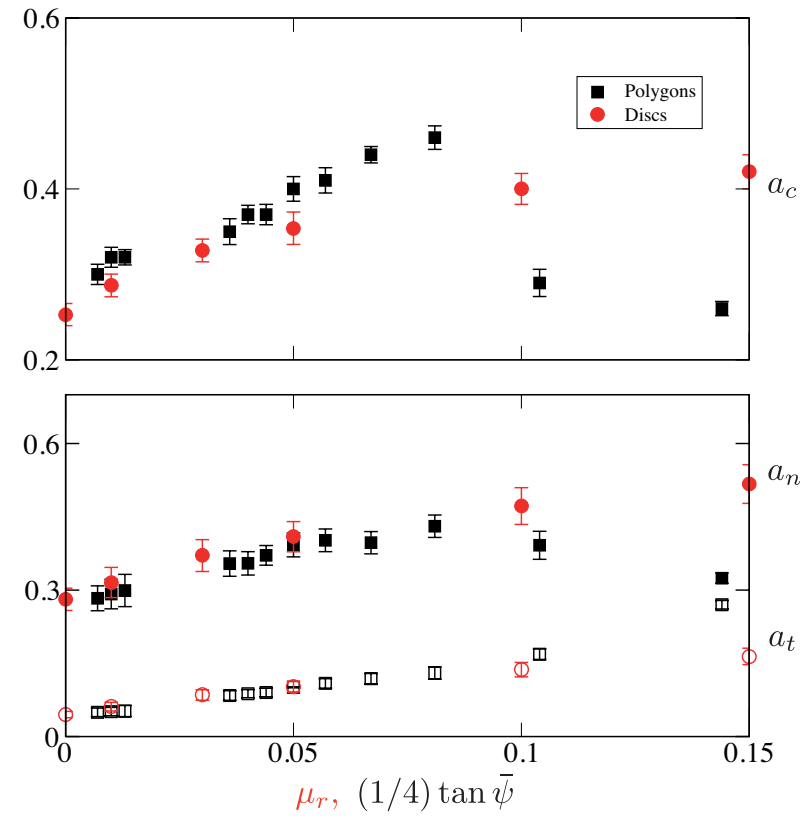

FIGURE 5. Contact anisotropy $a_{c}$ (Up) and force anisotropies, $a_{n}$ (full symbols) and $a_{t}$ (empty symbols) (Down), as functions of $\mu_{r}$ for the disks and of $(1 / 4) \tan \bar{\psi}$ for the polygons. Error bars indicate the standard deviation.

\section{CONCLUSION}

To sum up, the simulations presented in this article provide strong evidence for the mapping between the two studied parameters, i.e., rolling resistance and shape angularity. This correspondence was established by considering shear strength, solid fraction, force and fabric anisotropies, and the PDFs of normal forces in the steady state. A practical consequence of this finding is that rolling resistance may be employed to imitate the effect of angular shape in discrete-element simulations of granular materials. More importantly, it suggests that the hindrance of particle rotations is a major effect of angular particle shape.

\section{ACKNOWLEDGMENTS}

This work was financially supported by the FranceColombia Ecos-Nord project.

\section{REFERENCES}

1. J. Ai, J.-F. Chen, J. M. Rotter, and J. Y. Ooi, Powder Technology 206, 269-282 (2011).

2. K. Iwashita, and M. Oda, Journal of Engineering Mechanics 124, 285-292 (1998).
3. J.-Y. Delenne, M. S. El Youssoufi, F. Cherblanc, and J.-C. Bénet, Int. J. Numer. Anal. Meth. Geomech. 28, 1577-1594 (2004).

4. F. Calvetti, and R. Nova, Powders and Grains Conference Proceedings Vol. 1, 245-249 (2005).

5. M. J. Jiang, H. S. Yu, and D. Harris, Int. J. Numer. Anal. Meth. Geomech. 30, 723-761 (2006).

6. N. Estrada, E. Azéma, F. Radjai, and A. Taboada, Phys. Rev. E 84, 011306 (2011).

7. A. Taboada, K.-J. Chang, F. Radjai, and F. Bouchette, $J$. Geophys. Res. 110, B09202 (2005).

8. J. J. Moreau, European Journal of Mechanics, A/Solids 13 (Suppl.), 93-114 (1994).

9. M. Jean, Mechanics of Geometrical Interfaces, Elsevier, New York, 1995, pp. 463-486.

10. M. Jean, Comput. Methods Appl. Mech. Engrg 117, 235 257 (1999).

11. F. Radjai, and V. Richefeu, Mechanics of Materials 41, $715-728$ (2009).

12. N. Estrada, A. Taboada, and F. Radjaï, Phys. Rev. E 78, 021301 (2008).

13. G. Saussine, C. Cholet, P. Gautier, F. Dubois, C. Bohatier, and J. Moreau, Comput. Methods Appl. Mech. Eng. 195, 2841 - 2859 (2006).

14. F. Radjai, D. E. Wolf, M. Jean, and J.-J. Moreau, Phys. Rev. Lett. 80, 61-64 (1998).

15. T. Matsushima, and R. Nova, Powders and Grains Conference Proceedings Vol. 1, 1319-1323 (2005).

16. L. Rothenburg, and R. J. Bathurst, Geotechnique 39, 601-614 (1989).

17. F. Radjai, M. Jean, J.-J. Moreau, and S. Roux, Phys. Rev. Lett. 77, 274- (1996). 